\title{
IL-8 induces the epithelial-mesenchymal transition of renal cell carcinoma cells through the activation of AKT signaling
}

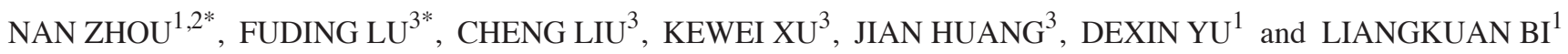 \\ Departments of ${ }^{1}$ Urology and ${ }^{2}$ Ultrasound, The Second Affiliated Hospital of Anhui Medical University, Hefei, Anhui 230601; \\ ${ }^{3}$ Department of Urology, Sun Yat-sen Memorial Hospital, Sun Yat-sen University, Guangzhou, Guangdong 510120, P.R. China
}

Received April 6, 2015; Accepted June 16, 2016

DOI: $10.3892 / 01.2016 .4900$

\begin{abstract}
The epithelial-mesenchymal transition (EMT) process has increasingly been examined due to its role in the progression of human tumors. Renal cell carcinoma (RCC) is one of the most common urological tumors that results in patient mortality. Previous studies have demonstrated that the EMT process is closely associated with the metastasis of RCC; however, the underlying molecular mechanism has not been determined yet. The present study revealed that interleukin (IL)-8 was highly expressed in metastatic RCC. IL-8 could induce the EMT of an RCC cell line by enhancing $\mathrm{N}$-cadherin expression and decreasing E-cadherin expression. Furthermore, IL- 8 could induce AKT phosphorylation, and the phosphatidylinositol-4,5-bisphosphate 3-kinase inhibitor LY294002 could inhibit the EMT of RCC cells that was induced by IL- 8 . Therefore, these results suggest that IL-8 is able to promote the EMT of RCC through the activation of the AKT signal transduction pathway, and this may provide a possible molecular mechanism for RCC metastasis.
\end{abstract}

\section{Introduction}

Renal cell carcinoma (RCC) is one of the most common tumors of the urological system in humans (1). Treatment for this disease is difficult due to its high metastatic tendency, as well as its resistance to radiotherapy and chemotherapy (2). Patients with RCC develop metastasis in $\sim 33 \%$ of cases (3), which causes $\sim 35 \%$ of mortality (4-6). Recently, patients with RCC are being diagnosed at increasingly younger ages (7). Approximately $20 \%$ of patients with RCC lose the possibility of radical treatment approaches due to metastasis $(3,8)$. Furthermore, $40-50 \%$ of those patients with localized advanced disease will

Correspondence to: Professor Liangkuan Bi, Department of Urology, The Second Affiliated Hospital of Anhui Medical University, 678 Furong Road, Hefei, Anhui 230601, P.R. China E-mail: blk14@126.com

${ }^{*}$ Contributed equally

Key words: renal cell carcinoma, epithelial-mesenchymal transition, interleukin-8, AKT, metastasis ultimately progress to metastatic disease (9). Therefore, it is important to determine the molecular mechanism of invasion and metastasis in RCC.

The epithelial-mesenchymal transition (EMT) is an important process in tumor metastasis (10). Tumor EMT is a phenotypic switch that promotes the acquisition of a fibroblastoid-like morphology by epithelial tumor cells, increased expression of mesenchymal-associated proteins, decreased expression of epithelial markers, and enhanced tumor cell motility and invasiveness (11-13). A previous study demonstrated that EMT contributes to the metastasis of RCC (14). However, the underlying cellular and molecular mechanisms have not been clarified yet.

As a critical chemoattractant, interleukin (IL)- 8 is known to participate in cancer progression (15). In recent years, an association between IL-8, tumor EMT and tumor stemness has been demonstrated $(11,13,16)$. Previous research has indicated that renal cancer cells in vitro are able to secrete IL-8, particularly those cell lines that are undergoing EMT and have metastatic potential (17). However, the role of IL-8 in renal cancer progression and in the induction of EMT in RCC remain unknown. The serine-threonine kinase AKT has been demonstrated to participate in signal transmission pathways in numerous types of cancer (18). The potential role of the activation of AKT in RCC remains unclear. The present study aimed to identify the potential role of IL-8 as well as that of AKT activation in RCC to demonstrate a possible molecular mechanism for RCC metastasis.

\section{Materials and methods}

Materials. The renal carcinoma 786-O cell line was purchased from the American Type Culture Collection (Manassas, VA, USA). IL-8 and Super ECL Plus hypersensitivity light-emitting solution were purchased from Sigma-Aldrich (St. Louis, MO, USA). Anti-IL-8 antibody was purchased from Abnova (rabbit; polyclonal; catalog no. ABIN453704; Taipei City, Taiwan), while anti-phospho-AKT (mouse; monoclonal; catalog no. 12694) and anti-AKT (rabbit; monoclonal; catalog no. 4691) antibodies were purchased from Cell Signaling Technology, Inc. (Danvers, MA, USA) (all 1:1,000 dilution). Anti- $\beta$-actin (mouse; monoclonal; catalog no. sc8432), anti-E-cadherin (mouse; monoclonal; catalog no. sc8426) and anti-N-cadherin (mouse; monoclonal; catalog no. sc8424) 
antibodies were purchased from Santa Cruz Biotechnology, Inc. (Dallas, TX, USA) (all 1:1,000 dilution). LY294002 (S1737), a phosphatidylinositol-4,5-bisphosphate 3-kinase (PI3K) inhibitor, was purchased from Beyotime Institute of Biotechnology (Haimen, China). RPMI 1640 was purchased from Invitrogen (Thermo Fisher Scientific, Inc., Waltham, MA, USA). Fetal bovine serum (FBS) was purchased from HyClone (GE Healthcare Life Sciences, Logan, UT, USA). The culture plates were purchased from Corning Incorporated (Corning, NY, USA). Phenylmethane sulfonyl fluoride and bovine serum albumin were purchased from Gen-View Scientific Inc. (El Monte, CA, USA). Polyvinylidene fluoride membranes were purchased from EMD Millipore (Billerica, MA, USA). Western blot and immunoprecipitation cell lysates were prepared in the laboratory.

Cell culture. The human RCC 786-O cell line was maintained in RPMI 1640 medium supplemented with 10\% FBS at $37^{\circ} \mathrm{C}$ and $5 \% \mathrm{CO}_{2}$. To determine the cellular growth curve, $5 \times 10^{4}$ cells suspended in $2 \mathrm{ml}$ medium were seeded into a 6 -well plate and cultured under normal conditions. At 24 or $48 \mathrm{~h}$ after seeding, the cells in each well were trypsinized and counted.

Clinical data and renal cancer tissues. A total of 20 fresh RCC tissues and their corresponding paired adjacent non-cancerous tissue samples were randomly selected from patients undergoing laparoscopic radical nephrectomy at the Sun Yat-sen Memorial Hospital (Guangzhou, China) from January 2009 to December 2011. The tissues were collected and processed immediately within $15 \mathrm{~min}$. Each sample was frozen and stored at $-80^{\circ} \mathrm{C}$. The paired non-cancerous tissues were isolated from $\geq 1 \mathrm{~cm}$ away from the tumor border and were demonstrated to lack tumor cells by microscopy. All patients in the present study met the following inclusion criteria: The resected mass was identified as RCC by pathological examination; no anti-cancer treatments were administered prior to surgery; and complete resection of all tumor nodules was verified by the cut surface being free of cancer by pathological examination. Enzyme-linked immunosorbent assay was performed to detect the supernatant prepared for determining the IL-8 according to the manufacturer's protocol (Bray Leino Group Ltd., Chicago, IL, USA).

IL-8-mediated induction of EMT in 786-O cells. 786-O cells were cultured for $24 \mathrm{~h}$ in RPMI 1640 containing 10\% FBS at $37^{\circ} \mathrm{C}$ and $5 \% \mathrm{CO}_{2}$. When the cells reached a density of $30-50 \%$, the medium was replaced with serum-free medium for $12 \mathrm{~h}$. Subsequently, the medium was replaced again in the experimental group, which had IL-8 added at a concentration of $100 \mu \mathrm{g} / 1$, whereas the control group had normal medium culture without additional IL- 8 . In accordance with the experimental design, cells were collected at $96 \mathrm{~h}$ after the follow-up test, as shown in Fig. 1.

Migration and invasion assays. Migration and invasion assays were performed as described in the BD Biosciences Operations Guide (19). Briefly, $100 \mu 1$ Matrigel was added to the upper filters of the cell culture inserts, and immediately placed in a culture plate. Subsequently, the $786-\mathrm{O}$ cell density was
A

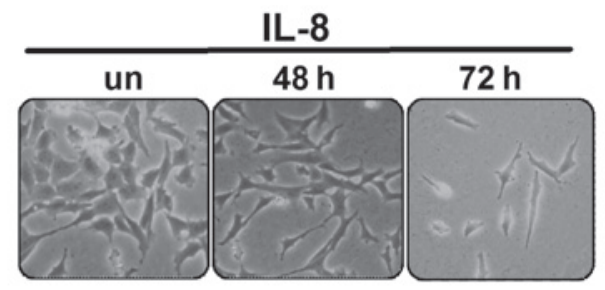

B

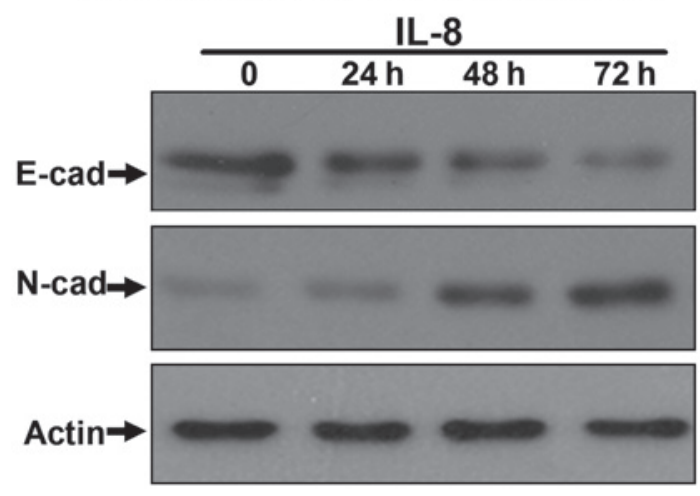

Figure 1. IL-8 promotes epithelial-mesenchymal transition in renal cell carcinoma cells. (A) Morphology of 786-O cells in the IL-8-treated and untreated groups. Photomicrographs were acquired at x100 magnification. (B) Immunoblots were performed with antibodies against the indicated antigens in whole-cell lysates. The expression of the E-cadherin epithelial cell marker and the $\mathrm{N}$-cadherin mesenchymal cell marker were examined in 786-O cells following stimulation with IL-8. IL-8, interleukin-8; un, untreated; cad, cadherin.

adjusted to $5 \times 10^{5}$ cells/ml. RPMI 1640 medium with or without IL-8 was then added to the lower filters, and $1 \times 10^{5}$ cells were added into the upper filters and incubated for the indicated time. The migrated or invaded cells in the lower filters were fixed and counted under a microscope.

Proliferation assay. 786-O cells were collected, and the cell suspension was adjusted to a concentration of $5 \times 10^{4}$ cells $/ \mathrm{ml}$. The cell culture medium, with or without IL-8, was added to the experimental and control treatment groups, and incubated for the indicated time. Subsequently, $20 \mu 1$ 3-(4,5-dimethylthiazol-2-yl)-2,5-diphenyltetrazolium bromide (MTT) solution was added into each well and later removed. Dimethyl sulfoxide was then added into each hole of the 96-well plates, incubated for $10 \mathrm{~min}$, and the absorbance was detected in a microplate reader immediately after the crystals were fully dissolved.

Western blotting. MSCs at $\sim 75 \%$ confluence were harvested and the cell density was adjusted to $1 \times 10^{6} / \mathrm{ml}$. The cell suspension was added to 6 -well plates ( $3 \mathrm{ml} /$ well, $3 \times 10^{6} /$ well). The cells were maintained in serum-free medium overnight. In the IL-8 group, the cells were treated with IL-8 for 15, 30 and $60 \mathrm{~min}$, while in the anti-CXCR2 group, the cells were pretreated with anti-CXCR2 antibodies for $30 \mathrm{~min}$ and then with IL-8 for 15, 30 and $60 \mathrm{~min}$. Next, these cells were washed twice with cold phosphate-buffered saline and were transferred into centrifuge tubes, followed by centrifugation at $1,800 \mathrm{x} \mathrm{g}$ for $4 \mathrm{~min}$. The supernatant was removed. The cells were mixed with lysis buffer $(80 \mu \mathrm{l})$ and pipetted. The lysate was kept on ice for $15 \mathrm{~min}$, followed by centrifugation at $12,000 \mathrm{x} \mathrm{g}$ at $4^{\circ} \mathrm{C}$ for $10 \mathrm{~min}$. The supernatant was collected $(60 \mu \mathrm{l})$ and mixed with 
A

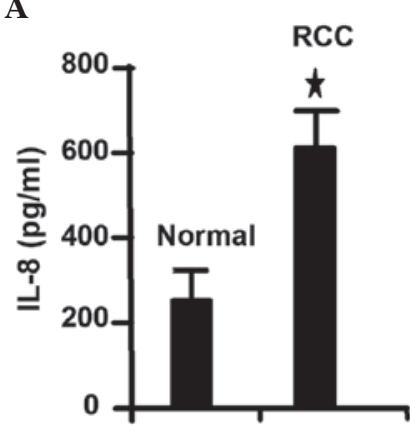

B

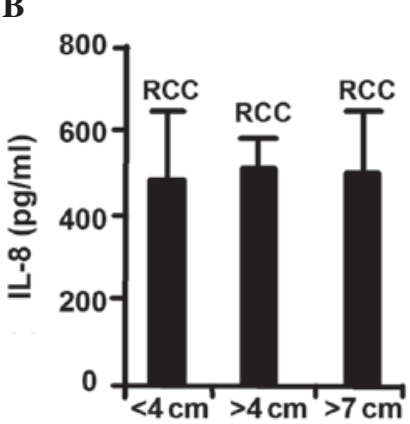

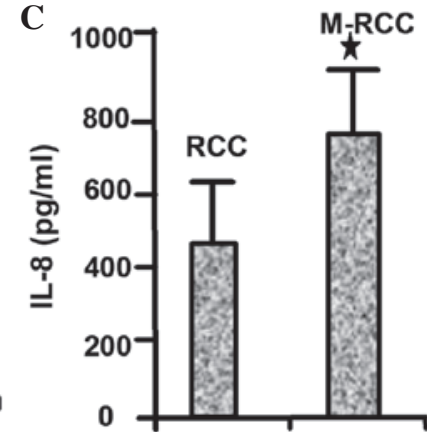

Figure 2. IL-8 expression level correlates with the metastatic ability and the staging of RCC, but not with the size of renal tumors. (A) IL-8 protein level in the RCC tissue compared with that in the normal tissue in the kidney. ${ }^{*} \mathrm{P}<0.05$ vs. normal tissue. (B) Protein levels of IL- 8 in the different tumor sizes, as determined by ELISA. (C) Expression of IL-8 in RCC and metastatic RCC, as determined by ELISA. *P<0.05 vs. RCC. IL- 8 , interleukin-8; RCC, renal cell carcinoma; M-RCC, metastatic renal cell carcinoma; ELISA, enzyme-linked immunosorbent assay.

$4 \%$ sodium dodecyl sulfate $(20 \mu \mathrm{l})$, followed by heating at $95^{\circ} \mathrm{C}$ for 5 min. Proteins were subjected to $12 \%$ sodium dodecyl sulfate-polyacrylamide gel electrophoresis and then were transferred onto a polyvinyldifluoride (PVDF) membrane. The PVDF membrane was blocked in 5\% skimmed milk in $1 \mathrm{X}$ Tris-buffered saline containing $0.05 \%$ Tween-20 (TBST) at room temperature for $60 \mathrm{~min}$. The membrane was incubated with the corresponding antibody (dilution, 1:1,000) at $4^{\circ} \mathrm{C}$ overnight (10 $\mathrm{ml}$ of $1 \mathrm{X}$ TBST, $0.5 \mathrm{~g}$ bovine serum albumin, and $10 \mu \mathrm{l}$ of $1-\mathrm{mg} / \mathrm{ml}$ antibody). The membrane was washed with TBST and then treated with horseradish peroxidase-conjugated goat anti-rabbit secondary antibody (dilution, 1:5,000) at room temperature for $1 \mathrm{~h}$. Subsequent to washing in TBST, visualization was performed with an enhanced chemiluminescence kit, and bands were observed with a gel image system. Similar procedures were employed to detect the expression of $\beta$-actin. The expression of target proteins was normalized to that of $\beta$-actin.

Statistical analysis. Experimental data are presented as the mean \pm standard deviation. SPSS 13.0 software (SPSS, Inc., Chicago, IL, USA) was used for statistical analysis. Student's $t$ test was used to compare two independent groups of data. $\mathrm{P}<0.05$ was considered to indicate a statistically significant difference.

\section{Results}

IL-8 is highly expressed in RCC. In several tumor types, including lung, colon and breast cancer, the expression of IL-8 is elevated in tumor tissues compared with normal tissues (20,21). In agreement with these results, the present study revealed that IL-8 is highly expressed in RCC compared with normal tissues (Fig. 2A). Notably, high IL-8 expression was not correlated with the size of the tumor (Fig. 2B). In addition, the present study revealed that IL-8 is highly expressed in metastatic RCC (Fig. 2C). These results demonstrated that IL-8 expression level is associated with the metastatic ability of RCC cells.

IL-8 can induce EMT in RCC cells. Tumor EMT involves a phenotypic switch that promotes the acquisition of a fibroblastoid-like morphology by epithelial tumor cells, and it is an important step for cancer cells to acquire metastatic capability $(11,22)$. To study the potential role of IL-8 in the metastatic ability of RCC, the induction of RCC EMT induced by IL- 8 was tested. The $786-\mathrm{O}$ cells at $\sim 75 \%$ confluence were divided into two groups, the control group (cultured without IL-8) and the IL-8-treated group (experimental group). As shown in Fig. 1A, after being cultured for the indicated time, the morphology of the 786-O cells in the IL-8 group had changed, switching from epithelial tumor cell morphology to fibroblastoid-like morphology, and reducing cell polarity and cell-to-cell contacts. In contrast, the morphology of 786-O cells cultured without IL-8 remained unchanged. Western blot analyses demonstrated reduced expression of the E-cadherin epithelial marker and upregulation of the $\mathrm{N}$-cadherin mesenchymal marker in the IL-8-stimulated 786-O cells (Fig. 1B), which is the most important characteristic of cancer cells undergoing EMT (23). These observations suggested that IL-8 may aid 786-O cells to reduce their polarity, enhance their motility and acquire a fibroblastoid-like morphology. Furthermore, IL- 8 was able to upregulate mesenchymal markers expression and downregulate epithelial markers expression in these cells, indicating that IL- 8 can induce EMT in RCC cells.

IL-8 promotes migration and invasion of RCC cells without affecting cell proliferation. Tumor cells undergoing EMT reduce their polarity and cell-to-cell contacts, and increase their motility and invasiveness, which may be important in tumor metastasis and progression (24). To determine the effect of IL-8 on 786-O cell migratory ability and invasiveness propensity, migration and invasion assays were used. As shown in Fig. 3A and B, the quantity of cells that penetrated through the matrigel was much larger in the IL-8-stimulated group than in the normal group. However, the cellular growth was not influenced when evaluated by MTT assay (Fig. 3C). These results demonstrated that IL- 8 can promote RCC cells migration and invasion, and as a result, it may also promote RCC metastasis, although it had no effect on the proliferation of RCC cells.

IL-8 can enhance the levels of phosphorylated AKT in RCC cells. The PI3K/AKT is an important signaling pathway and correlates with the progression of multiple malignant tumor types (25). AKT, also called protein kinase B, is regarded as 

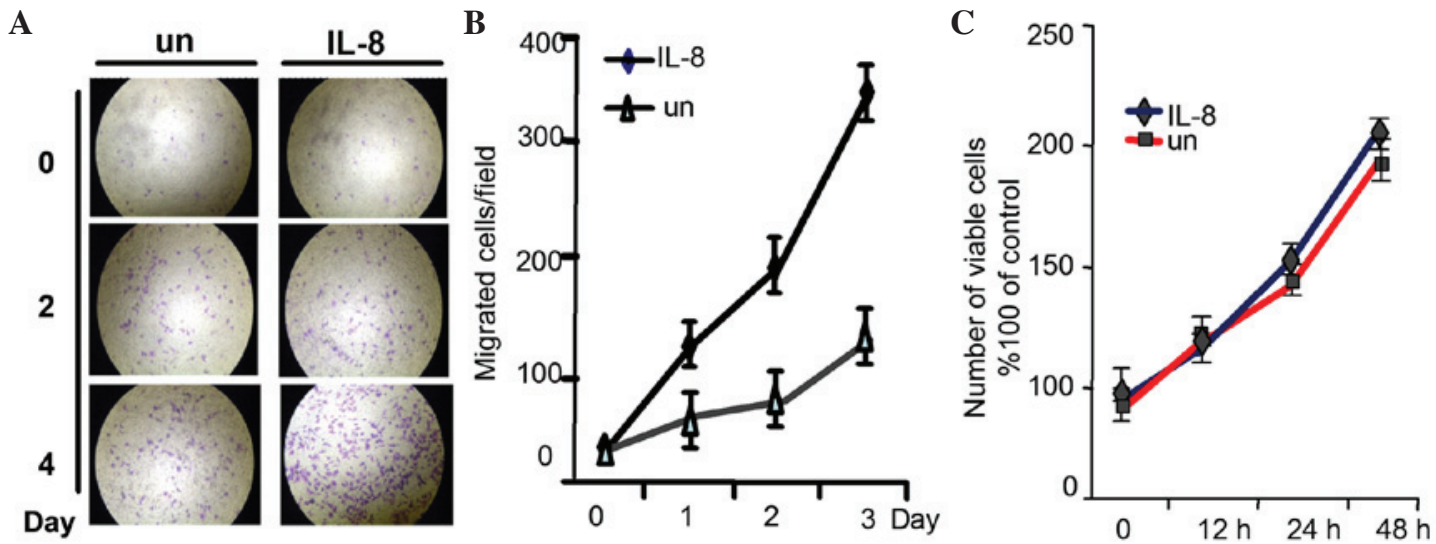

Figure 3. IL-8 promoted the migration and invasion of renal cell carcinoma cells. (A) The migratory and invasive abilities of 786-O cells stimulated with IL-8 or not were evaluated by Transwell assays. Photomicrographs were obtained at x100 magnification. (B) Number of migrated 786-O cells/field in the IL-8-treated and untreated groups. (C) Number of viable 786-O cells/field in the IL-8-treated and untreated groups, as evaluated by 3-(4,5-dimethylthiazol-2-yl)-2,5-diphenyltetrazolium bromide assay. IL-8, interleukin-8; un, untreated.

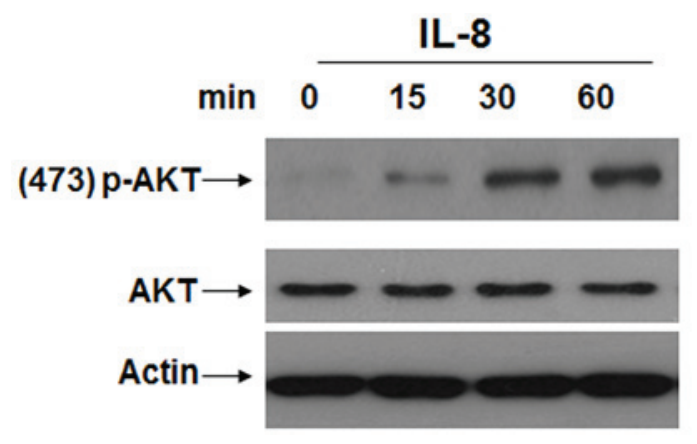

Figure 4. IL-8 promotes AKT activation in renal cell carcinoma cells. Immunoblots of whole-cell lysates following IL-8 stimulation for different time periods showing the levels of phosphorylated and total AKT. IL-8, interleukin-8; p-AKT, phosphorylated AKT.

the most important downstream signaling molecule of PI3K, and is activated following phosphorylation (26). According to recent research, the PI3K/AKT signaling pathway is involved in EMT of numerous tumor cells $(27,28)$. To study the mechanism by which IL-8 promotes EMT in RCC, the levels of phosphorylated AKT were examined in 786-O cells following stimulation with different concentrations of IL-8. It was observed that appropriate IL-8 stimulation $(100 \mu \mathrm{g} / \mathrm{l})$ for 15,30 or 60 min could noticeably enhance the levels of phosphorylated AKT (Fig. 4). These results suggested that IL-8 may be a critical factor to activate AKT, and this may be one of the important mechanisms for regulating EMT of RCC cells.

IL-8 participates in regulating the EMT of RCC cells through the activation of AKT signaling. As mentioned above, IL- 8 can elevate the phospho-AKT level, which activates the PI3K/AKT signaling pathway. Consequently, IL-8 may promote the migration and invasion of RCC cells. To further examine the role of IL-8 in regulating the EMT of RCC, LY294002 was used to inhibit AKT activation. The induction of EMT caused by IL-8 was suppressed by LY294002 (Fig. 5). These results indicate that AKT signaling is essential for IL-8 promotion of cellular motility in RCC cells.
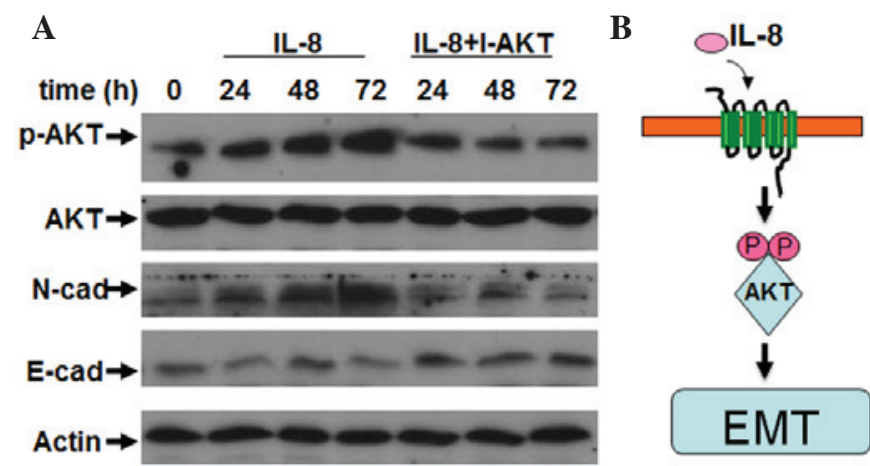

Figure 5. IL-8 promotes RCC cell EMT through AKT activation. (A) Immunoblots of whole-cell lysates using an anti-AKT (pan) antibody upon stimulation with IL-8. RCC EMT was evaluated by detecting the expression of E-cadherin and $\mathrm{N}$-cadherin in 786-O cells. $\beta$-actin served as the loading control. (B) Possible mechanism by which IL-8 induces the EMT of RCC cells. IL-8, interleukin-8; p-AKT, phosphorylated AKT; cad, cadherin; EMT, epithelial-mesenchymal transition; I-AKT, AKT inhibitor; $\mathrm{RCC}$, renal cell carcinoma.

\section{Discussion}

The metastasis of RCC remains an intractable problem in the clinic, mainly because it is not known exactly how RCC cells acquire their invasive ability (29). The present study demonstrated that IL-8 was highly expressed in metastatic RCC and it could induce the EMT of RCC cells, which was associated with AKT phosphorylation, and suggested that IL-8 may induce the EMT of RCC by AKT signaling activation. This may be a potential mechanism for RCC metastasis.

Malignant tumor progression consists of distinct steps, including tumor growth, angiogenesis and EMT (30). RCC is one of the most common malignant tumors of the urinary system, and the main obstacle that remains in the current clinical management is its metastatic propensity and resistance to chemotherapy, radiotherapy and immunological therapy (31). Multiple studies have revealed that EMT induction in human carcinoma cells is closely associated with the enhanced secretion of numerous cytokines and chemokines, as well as with the metastasis of several solid carcinomas, including 
renal cancer $(32,33)$. However, the exact mechanism of these pathological processes is not clear. Elucidating the mechanism underlying RCC metastasis, which could lead to improved treatments, is of considerable significance.

IL-8 is a key chemokine that is important in inflammation and cancer (34). Increasing evidence in the literature has supported the role of IL-8 as a critical factor for tumor growth, angiogenesis, metastasis and development of tumors (35). Furthermore, it may be the connecting factor between tumor cells and the tumor microenvironment. Tumor cells tend to undergo EMT within the tumor microenvironment, and then gain motility and invasive properties, and IL-8 is considered to play a significant role in this process (36). In melanoma, tumor-derived IL- 8 has been demonstrated to promote tumor cell proliferation, survival and migration via its autocrine activity (34). The present study revealed that IL- 8 was highly expressed in metastatic RCC cells, and was able to promote $786-\mathrm{O}$ cell migration and invasion. These results suggest an important potential mechanism for renal cancer metastasis, and IL- 8 may be a potential drug target for preventing and inhibiting RCC metastasis.

In recent years, the importance of EMT in the progression of carcinomas has been demonstrated. It has been revealed that tumor cells undergoing EMT have the potential to reduce the expression of basement membrane constituents (collagen type IV and laminins) and to augment the secretion of extracellular matrix constituents (osteonectin and collagen type I) (37). Consequently, tumor epithelial cells actively downregulate cell-cell adhesion systems, lose polarity and acquire a mesenchymal phenotype with reduced intercellular interactions (38). In the present study, the motility and invasiveness of 786-O RCC cells undergoing EMT were noticeably enhanced, and this phenomenon was positively correlated with IL-8. Upon IL-8 stimulation, 786-O cells reduced the expression of epithelial markers (E-cadherin) and upregulated the expression of mesenchymal markers ( $\mathrm{N}$-cadherin), acquired a phenotypic switch of EMT, and improved their ability to penetrate through matrigel. The present data demonstrated that IL- 8 could induce EMT in renal cancer cells and may promote RCC metastasis.

Activation of the PI3K signaling pathway is highly prevalent in tumor growth, and it serves as a relay where signals that emanate from the cell membrane are received and are converted into intracellular signals that promote proliferation and survival (39). The phospho-AKT level is a classic indicator to evaluate AKT activity (40). Okui et al demonstrated that the PI3K/AKT signaling pathway is closely associated with EMT induction and human tumor development (41). In the present study, IL-8 stimulation could noticeably enhance the phosphorylated AKT levels as well as the motility and invasiveness of RCC cells. Furthermore, when AKT signaling was blocked using LY294002 to inhibit AKT activation, the induction of EMT mediated by IL- 8 stimulation could be eliminated. This observation indicated that AKT is a key signaling pathway by which IL-8 regulates the migratory and invasive abilities of RCC cells. Taken together, these data indicate that IL-8 may induce EMT of RCC through the activation of AKT signaling.

In conclusion, the results of the present study demonstrated that IL-8 is highly expressed in metastatic RCC, and it can promote 786-O cell migration and invasion by inducing EMT via the activation of AKT signaling. However, there are various other signaling pathways such as the nuclear factor- $\kappa \mathrm{B}$ signaling pathway that also are important in the induction and maintenance of EMT (42). The impact that EMT plays on tumor metastasis is still controversial (43-45). To better understand the exact mechanisms of tumor metastasis and its association with EMT and IL-8, further studies are required to characterize other signaling pathways, as well as the phenotype of RCC cells, in the future.

\section{Acknowledgements}

The present study was supported by the National Natural Science Foundation of China (Beijing, China; grant no. 81572507), and the Natural Science Foundation of Anhui Province (grant no. $1508085 \mathrm{SQH} 225)$.

\section{References}

1. Moch H: An overview of renal cell cancer: Pathology and genetics. Semin Cancer Biol 23: 3-9, 2013.

2. Pecuchet N, Fournier LS and Oudard S: New insights into the management of renal cell cancer. Oncology 84: 22-31, 2013.

3. Flanigan RC, Campbell SC, Clark JI, Picken MM and Picken MM: Metastatic renal cell carcinoma. Curr Treat Options Oncol 4: 385-390, 2003.

4. Yao X, Qi L, Chen X, Du J, Zhang Z and Liu S: Expression of CX3CR1 associates with cellular migration, metastasis, and prognosis in human clear cell renal cell carcinoma? Urol Oncol 32: 162-170, 2014.

5. Escudier B, Albiges L and Sonpavde G. Optimal management of metastatic renal cell carcinoma: Current status. Drugs 73: 427-438, 2013.

6. Sun M, Shariat SF, Trinh QD, Meskawi M, Bianchi M, Hansen J, Abdollah F, Perrotte P and Karakiewicz PI: An evidence-based guide to the selection of sequential therapies in metastatic renal cell carcinoma. Ther Adv Urol 5: 121-128, 2013.

7. Washio M, Mori M, Mikami K, Miki T, Watanabe Y, Nakao M, Kubo T, Suzuki K, Ozasa K, Wakai K and Tamakoshi A: Risk factors for renal cell carcinoma in a Japanese population. Asian Pac J Cancer Prev 15: 9065-9070, 2014.

8. Syrios J, Kechagias G and Tsavaris N: Treatment of patients with metastatic renal cell carcinoma undergoing hemodialysis: Case report of two patients and short literature review. BMC Nephrol 14: 84, 2013.

9. Ljungberg B: The role of metastasectomy in renal cell carcinoma in the era of targeted therapy. Curr Urol Rep 14: 19-25, 2013.

10. Lin JC, Liao SK, Lee EH, Hung MS, Sayion Y, Chen HC, Kang CC, Huang LS and Cherng JM: Molecular events associated with epithelial to mesenchymal transition of nasopharyngeal carcinoma cells in the absence of Epstein-Barr virus genome. J Biomed Sci 16: 105, 2009.

11. Palena C, Hamilton DH and Fernando RI: Influence of IL-8 on the epithelial-mesenchymal transition and the tumor microenvironment. Future Oncol 8: 713-722, 2012.

12. Bates RC, DeLeo MJ III and Mercurio AM: The epithelial-mesenchymal transition of colon carcinoma involves expression of IL-8 and CXCR-1-mediated chemotaxis. Exp Cell Res 299: 315-324, 2004.

13. Esteban MA, Bao X, Zhuang Q, Zhou T, Qin B and Pei D: The mesenchymal-to-epithelial transition in somatic cell reprogramming. Curr Opin Genet Dev 22: 423-428, 2012.

14. Mikami S, Oya M, Mizuno R, Kosaka T, Katsube K and Okada Y: Invasion and metastasis of renal cell carcinoma. Med Mol Morphol 47: 63-67, 2014.

15. Todorović-Raković N and Milovanović J: Interleukin-8 in breast cancer progression. J Interferon Cytokine Res 33: 563-570, 2013.

16. Prud'homme GJ: Cancer stem cells and novel targets for antitumor strategies. Curr Pharm Des 18: 2838-2849, 2012.

17. Huang D, Ding Y, Zhou M, Rini BI, Petillo D, Qian CN, Kahnoski R, Futreal PA, Furge KA and Teh BT: Interleukin-8 mediates resistance to antiangiogenic agent sunitinibin renal cell carcinoma. Cancer Res 70: 1063-1071, 2010.

18. Radisavljevic Z: AKT as locus of cancer angiogenic robustness and fragility. J Cell Physiol 228: 21-24, 2013. 
19. Hasan MK, Nafady A, Takatori A, Kishida S, Ohira M, Suenaga Y Hossain S, Akter J, Ogura A, Nakamura Y, et al: ALK is regulates a MYCN target gene and cell migration and invasion in neuroblastoma. Sci Rep 3: 3450, 2013.

20. Bai Z, Tai Y, Li W, Zhen C, Gu W, Jian Z, Wang Q, Lin JE, Zhao Q, Gong W, et al: Gankyrin activates IL-8 to promote hepatic metastasis of colorectal cancer. Cancer Res 73 4548-4558, 2013.

21. Umekawa K, Kimura T, Kudoh S, Suzumura T, Oka T, Nagata M, Mitsuoka S, Matsuura K, Nakai T, Yoshimura N, et al: Plasma RANTES, IL-10 and IL-8 levels in non-small-cell lung cancer patients treated with EGFR-TKIs. BMC Res Notes 6: 139, 2013.

22. Zhu C, Li J, Cheng G, Zhou H, Tao L, Cai H, Li P, Cao Q, Ju X, Meng X, et al: miR-154 inhibits EMT by targeting HMGA2 in prostate cancer cells. Mol Cell Biochem 379: 69-75: 2013.

23. Gheldof A and Berx G: Cadherins and epithelial-to-mesenchymal transition. Prog Mol Biol Transl Sci 116: 317-336, 2013.

24. Simic P, Williams EO, Bell EL, Gong JJ, Bonkowski M and Guarente L: SIRT1 suppresses the epithelial-to-mesenchymal transition in cancer metastasis and organ fibrosis. Cell Rep 3: 1175-1186, 2013.

25. Carpenter RL and Jiang BH: Roles of EGFR, PI3K, AKT, and mTOR in heavy metal-induced cancer. Curr Cancer Drug Targets 13: 252-266, 2013.

26. Tong $X$ and Pelling JC: Targeting the PI3K/Akt/mTOR axis by apigenin for cancer prevention. Anticancer Agents Med Chem 13: 971-978, 2013.

27. Dobbin $\mathrm{Zc}$ and Landen $\mathrm{CN}$ : The Importance of the $\mathrm{PI} 3 \mathrm{~K} / \mathrm{AKT} / \mathrm{MTOR}$ pathway in the progression of ovarian cancer. Int J Mol Sci 14: 8213-8227, 2013.

28. Zhu H, Bhaijee F, Ishaq N, Pepper DJ, Backus K, Brown AS, Zhou X and Miele L: Correlation of Notch1, pAKT and nuclear $\mathrm{NF}-\kappa \mathrm{B}$ expression in triple negative breast cancer. Am J Cancer Res 3: 230-239, 2013

29. Larkin J, Swanton C and Pickering L: Optimizing treatment of metastatic renal cell carcinoma by changing mechanism of action. Expert Rev Anticancer Ther 11: 639-649, 2011.

30. Li F, Yin X, Luo X, Li HY, Su X, Wang XY, Chen L, Zheng K and Ren GS: Livin promotes progression of breast cancer through induction of epithelial-mesenchymal transition and activation of AKT signaling. Cell Signal 25: 1413-1422, 2013.

31. Gamelin E, Mertins SD, Regis JT, Mickley L, Abati A, Worrell RA, Linehan WM and Bates SE: Intrinsic drug resistance in primary and metastatic renal cell carcinoma. J Urol 162: 217-224, 1999

32. Soria G, Ofri-Shahak M,Haas I, Yaal-Hahoshen N,Leider-TrejoL, Leibovich-Rivkin T, Weitzenfeld P, Meshel T, Shabtai E, Gutman $\mathrm{M}$ and Ben-Baruch $\mathrm{A}$ : Inflammatory mediators in breast cancer: Coordinated expression of TNF $\alpha$ \& IL-1 $\beta$ with CCL2 \& CCL5 and effects on epithelial-to-mesenchymal transition. BMC Cancer 11: 130, 2011.

33. Chuang MJ, Sun KH, Tang SJ, Deng MW, Wu YH, Sung JS, Cha TL and Sun GH: Tumor-derived tumor necrosis factor-alpha promotes progression and epithelial-mesenchymal transition in renal cell carcinoma cells. Cancer Sci 99: 905-913, 2008.
34. Aggarwal BB, Shishodia S, Sandur SK, Pandey MK and Sethi G: Inflammation and cancer: How hot is the link? Biochem Pharmacol 72: 1605-1621, 2006.

35. Shen XH, Xu SJ, Jin CY, Ding F, Zhou YC and Fu GS: Interleukin-8 prevents oxidative stress-induced human endothelial cell senescence via telomerase activation. Int Immunopharmacol 16: 261-267, 2013.

36. Fernando RI, Castillo MD, Litzinger M, Hamilton DH and Palena C: IL-8 signaling plays a critical role in the epithelial-mesenchymal transition of human carcinoma cells. Cancer Res 71: 5296-5306, 2011.

37. Mathias RA, Chen YS, Wang B, Ji H, Kapp EA, Moritz RL, Zhu HJ and Simpson RJ: Extracellular remodelling during oncogenic Ras-induced epithelial-mesenchymal transition facilitates MDCK cell migration. J Proteome Res 9: 1007-1019, 2010.

38. Arima Y, Hayashi N, Hayashi H, Sasaki M, Kai K, Sugihara E, Abe E, Yoshida A, Mikami S, Nakamura S and Saya H: Loss of p16 expression is associated with the stem cell characteristics of surface markers and therapeutic resistance in estrogen receptor negative breast cancer. Int J Cancer 130: 2568-2579, 2012.

39. Juvekar A, and Wulf GM: Closing escape routes: Inhibition of IL-8 signaling enhances the anti-tumor efficacy of PI3K inhibitors. Breast Cancer Res 15: 308, 2013.

40. Kohn AD, Takeuchi F and Roth RA: Akt, a pleckstrin homology domain containing kinase, is activated primarily by phosphorylation. J Biol Chem 271: 21920-21926, 1996.

41. Okui G, Tobiume K, Rizqiawan A, Yamamoto K, Shigeishi H, Ono S, Higashikawa $\mathrm{K}$ and Kamata N: AKT primes snail-induced EMT concomitantly with the collective migration of squamous cells. J Cell Biochem 114: 2039-2049, 2013.

42. Grund EM, Kagan D, Tran CA, Zeitvogel A, Starzinski-Powitz A, Nataraja S and Palmer SS: Tumor necrosis factor-alpha regulates inflammatory and mesenchymal responses via mitogen-activated protein kinase kinase, p38, and nuclear factor kappaB in human endometriotic epithelial cells. Mol Pharmacol 73: 1394-1404, 2008

43. Li G, Zhang Y, Qian Y, Zhang H, Guo S, Sunagawa M, Hisamitsu T and Liu Y: Interleukin-17A promotes rheumatoid arthritis synoviocytes migration and invasion under hypoxia by increasing MMP2 and MMP9 expression through NF- $\mathrm{B} / \mathrm{HIF}-1 \alpha$ pathway. Mol Immunol 53: 227-236, 2013.

44. Kiefel H, Bondong S, Pfeifer M, Schirmer U, Erbe-Hoffmann N, Schäfer H, Sebens S and Altevogt P: EMT-associated up-regulation of L1CAM provides insights into L1CAM-mediated integrin signalling and $\mathrm{NF}-\mathrm{\kappa B}$ activation. Carcinogenesis 33 1919-1929, 2012.

45. Kang YH, Ji NY, Han SR, Lee CI, Kim JW, Yeom YI, Kim YH, Chun HK, Kim JW, Chung JW, et al: ESM-1 regulates cell growth and metastatic process through activation of NF- $\kappa \mathrm{B}$ in colorectal cancer. Cell Signal 24: 1940-1949, 2012. 